Proceedings of the 2012 Winter Simulation Conference

C. Laroque, J. Himmelspach, R. Pasupathy, O. Rose, and A. M. Uhrmacher, eds.

\title{
DESIGNING SERIOUS GAMES FOR REVENUE MANAGEMENT TRAINING AND STRATEGY DEVELOPMENT
}

\author{
Catherine Cleophas \\ Freie Universität Berlin \\ Garystr. 21 \\ D-14195 Berlin, GERMANY
}

\begin{abstract}
This paper proposes a framework for the design of serious games in the area of revenue management. At this time, there is little systematic consideration of simulation-based serious games and their set-up available in this field. The suggested framework regards games as structured in three layered stages and explicates decisions influencing their design and focus. These decisions are structured according to five aspects: concurrence, conditions, cognizance, cooperation and competition. Revenue management provides a particular challenge as its success depends not only on the customer choice, competition and sophisticated operations research algorithms, but also on analysts' understanding. Simulation systems are a well-known tool for the evaluation of revenue management approaches in research and practice and can easily be extended for use in serious games. The framework introduced here can be used for future evaluations of alternative designs of serious games aiming to improve revenue management understanding and strategy evaluation.
\end{abstract}

\section{INTRODUCTION}

In recent years, serious games have gained popularity as a novel approach to training and strategy development as documented for example by Michael and Chen (2005) and Charsky (2010). Serious games can take the form of participatory simulations with one or more human players or that of parallel challenges with achievements and an exploratory or competitive element. This paper considers both such types of serious games as they may be applied to revenue management.

Suppliers implementing revenue management strive to maximize overall revenue through the deliberate control of available combinations of product and price, exploiting the willingness to pay of different demand segments. The feasibility of demand segmentation represents one of the conditions of revenue management. Further conditions include both a fixed and limited capacity and a fixed and limited sales period. Based on these conditions, revenue management is applied mostly in service industries such as transport, hospitality and entertainment (see also (McMahon-Beattie and Yeoman 2011)).

Revenue management - see Talluri and van Ryzin (2004) for a thorough introduction - is a field that may provide novel challenges to the application of serious games. Training and strategy development for revenue management are complicated by elements of uncertainty such as demand and competitor behavior as well as by the complexity of the information systems used. Both types of challenges can be approached by serious games based on simulation systems: These can provide the opportunity to trace the results of automated systems under stable, reproducible conditions.

As will be shown in Section 2, the use of simulations of the evaluations of revenue management methods and strategies is widely accepted. According to Mukhopadhyay, Samaddar, and Colville (2007) and Currie and Rowley (2010), the success of revenue management depends not only on the mathematical methods implemented in the automated systems but also on the intuition and the training of analysts controlling and 


\section{Cleophas}

if necessary adjusting the results of these systems. For this reason, an extension of the use of simulations in revenue management to support serious games for training and strategy development seems only natural.

When serious games are considered in this paper, the definition by Michael and Chen (2005) is applied: Serious games are "games that do not have entertainment, enjoyment or fun as their primary purpose". In the context of revenue management, the primary purposes of serious games are training and evaluating and discussing alternative revenue management strategies.

To the author's knowledge, little literature introducing serious games for revenue management is available. This paper concentrates on formalizing the decisions that influence the set-up of serious games using such simulations.

In Section 2, relevant research in the areas of serious games, participatory simulation and revenue management is reviewed. As apparent from the consideration of competition and cooperation in the context of markets, the framework presented here focuses specifically on aspects relevant for revenue management. This paper views serious games for revenue management in three layered stages. The design of each stage is determined by a range of decisions based on five aspects - concurrence, conditions, cognizance, cooperation and competition. These aspects and the game design decisions they motivate are introduced in detail in Section 3. Subsequently, the consequence of alternative designs in the set-up, execution and analysis of games is explicated in Section 4. The resulting framework aims to provide a first basis for the systematic evaluation of different approaches to setting up, conducting and analyzing revenue management games with regard to diverse objectives. Finally, in Section 5, the key propositions of this paper are summarized. In this last section, the use of the proposed framework for the formulating hypotheses with regard to the effectiveness of revenue management games for training and strategy development is demonstrated, providing an outlook toward future research based on them.

\section{SIMULATION-BASED SERIOUS GAMES FOR REVENUE MANAGEMENT}

After introducing some basic terminology, this section focuses on existing research in the areas of simulationbased serious gaming and revenue management. For this purpose, the use of simulations for serious games is described first. Subsequently, literature detailing the use of simulations in the field of revenue management is summarized.

\subsection{Some Terminology}

Discussing simulation-based serious games for revenue management requires the clarification of some terminology. References to simulation modeling and simulation systems in this paper follow the definition by Law and Kelton (2000); both discrete-event and agent-based modeling approaches (see Law and Kelton (2000) and (Gilbert 2008) for introductions to the respective concepts) are applicable in the field of revenue management.

Simulations used for revenue management decision support are often of a stochastic nature: several major influencing factors of revenue management such as customer demand and competitor availabilities are not deterministic and therefore need to be modeled based on stochastic distributions (see Frank, Friedemann, and Schröder (2008)). As participatory simulations as described for example by Ramanath and Gilbert (2004) rely on human players taking on the role of one or more agents interacting in the simulation mode, they are frequently deterministic for practical reasons: Stochastic simulations require a large number of independent repetitions; it can be laborious to realize these repetitions in participatory games.

As described in the introduction, both participatory simulations and challenges including stochastic simulations as tools for strategy development end evaluation can be used for serious games. This paper focuses on serious games based on computer simulation systems, thereby neglecting alternative solutions such as paper-based war games and role playing.

When considering serious games making use of simulation systems, we differentiate two roles: players and trainers. Trainers are in charge of setting up games, moderating and controlling their execution and 


\section{Cleophas}

guiding the analysis of finished games. Players take part in games by analyzing information provided for them and choosing a set of actions that influence the game's outcome. In participatory simulations based on agent-based models, both trainers and players may take on the role of one or more agents during the game. Other agents may be controlled by automated algorithms implemented in the simulation system.

\subsection{Simulations for Serious Games}

As described for example by Halter (2006), the concept of "war games" has been around for a very long time. Building up on this, modern approaches to serious games based on simulations - as documented for example by Michael and Chen (2005) - enable the generation of interactive scenarios with emergent characteristics.

Whereas in traditional war games, all interacting parties had to be represented by human players, agentbased modeling offers ways of implementing artificial adversaries as described for instance by Ramanath and Gilbert (2004). Such partially or fully automated players introduce both benefits and challenges: As opposed to that of human game controllers, their behavior is not necessarily predetermined by the intentions or the explicit knowledge of experts setting up the game. As exemplified by experiments based on agentbased simulations, even experts may not always expect the macro-level consequences of micro-level rules of choice.

This is both a benefit and a challenge: Depending on the game objective, surprising and novel scenarios generated in this way may be more or less desirable. Depending on the implemented behaviors, automated players may be more or less realistic than players represented by trainers. Finally, when some players can be automated, the number of heterogeneous, independent parties is no longer limited by the number of actual participants.

However, the use for serious games is not reserved for participatory simulations. As mentioned for example by Gilbert (2008), simulations can also be used as a tool for the evaluation of alternative policies as well as for the exploration of a problem space. Both of these purposes allow for setting up serious games as a form of experimentation as suggested by Michael and Chen (2005).

Current literature lists a variety of tasks for which simulations - often combined with elements of virtual reality - can be employed for the purpose of serious games. These range from language education (e.g., (Johnson 2007)) over health care (see Kato (2010)) to the management of grocery stores as documented by Mathieu, Panzoli, and Picault (2011). All of these examples have in common that players are confronted with tasks and environments prepared by trainers. When players are evaluated on their performance, their success depends on the reaction of the simulation system to their input.

\subsection{Simulation Modeling for Revenue Management}

As in other fields where simulation methods can be applied, revenue management problems are frequently modeled using stochastic, discrete-event based simulations. The use of these simulations as described for example by Cleophas, Frank, and Kliewer (2009) allows to test the results of new methods and different parameter sets. In contrast to real-world evaluations, simulation modeling offers the opportunity of risk-free testing under stable and reproducible conditions. Both benefits play an important role with regard to revenue management, where real-world tests can be costly and their evaluation is difficult given shifting market conditions.

A general set of guidelines for revenue management simulations has first been provided by Frank, Friedemann, and Schröder (2008). The authors also hint at one of the major challenges of revenue management simulations, that of calibrating the customer model. As only limited information about demand is available, approximations of the quantity and the quality of demand and customer choice have to suffice.

As suggested for example by Hoang (2007), the focus of revenue management is shifting toward a customer-centric view, making its success dependent on the flexible choice of informed customers. This 


\section{Cleophas}

shift can be modeled in simulation systems through the inclusion of agent-based concepts as outlined by Cleophas (2012b).

Two examples of revenue management research supported by simulation modeling are (Abdelghany and Abdelghany 2007) and (Abdelghany and Abdelghany 2008). Prominent examples of airline revenue management simulators that are considered here in further detail are PODS and REMATE.

PODS (Passenger Origin Destination Simulator) has been designed by Boeing in 1995 (see Hopperstad (1995) and is used and maintained at the Massachusetts Institute of Technology (MIT). Based on work with PODS, a large body of research focusing mostly on the comparison of different methods for forecasting demand and optimizing availability controls has been published. One of the earliest publications based on results generated with PODS is (Belobaba and Wilson 1997).

The first version of REMATE (Revenue Management Training for Experts) has been introduced in 2009 by Lufthansa in cooperation with the Technical University Clausthal and University Paderborn and is used and extended in the course of further research projects for instance with Freie Universität Berlin. It implements a micro-level rational choice model of customer behavior with random elements. REMATE is applied for research as well, supporting the evaluation of revenue management methods as in the example provided by Gorin, Walczak, Bartke, and Friedemann (2012). As it has been created and is maintained in the industry, REMATE is not limited to research but is also applied for decision support and serious games as described by Cleophas (2012a).

It appears that the simulation-based serious games summarized here could provide a working template for the application of serious games for revenue management training and strategy development. However, the review of literature reveals few existing examples of serious games being implemented for this purpose. Only the examples documented by Puschel, Lang, Bodenstein, and Neumann (2010) and by Cleophas (2012a) indicate existing approaches: Whereas Puschel, Lang, Bodenstein, and Neumann (2010) uses the implementation as a serious game to realistically model consumer response and evaluate the success of policies, the simulation system described in Cleophas (2012a) compares the success of policies generated by human players given constant consumer demand.

\section{DESIGN ASPECTS OF REVENUE MANAGEMENT GAMES}

When a game is set up, conducted and its results are analyzed, the trainer in charge of the game has to consider several design decisions influencing the players' experience. The framework proposed by this paper structures these decisions according to five aspects that are to some degree conditional as shown in Figure 1.

Concurrence describes whether a game is played synchronously by several players; conditions include the information provided and the indicators used for performance evaluation; cognizance decisions consider the area of responsibility of each player; cooperation refers to the way in which both players in teams and teams of players work together; competition considers both the types of competition and the types of competitors included in a game. Each of these aspects is explained in detail based on examples from airline revenue management in the following sections.

\subsection{Concurrence: Synchronous or Asynchronous Game Play}

Game play for revenue management may be synchronous or asynchronous, implying concurrence and a lack thereof. Participatory games including several players have to include concurrence so that players can interact at the same time in the same simulation system. Participatory games including only a single human player as well as any game in which one player's actions do not influence the outcome of other players' actions do not necessitate concurrence, they may be played asynchronously.

Asynchronous games with only a single player can be participatory, if this player takes on the role of a component of the simulation model. This players' performance may still be evaluated as compared to the performance of other players if the other players were confronted with the same game at a different 


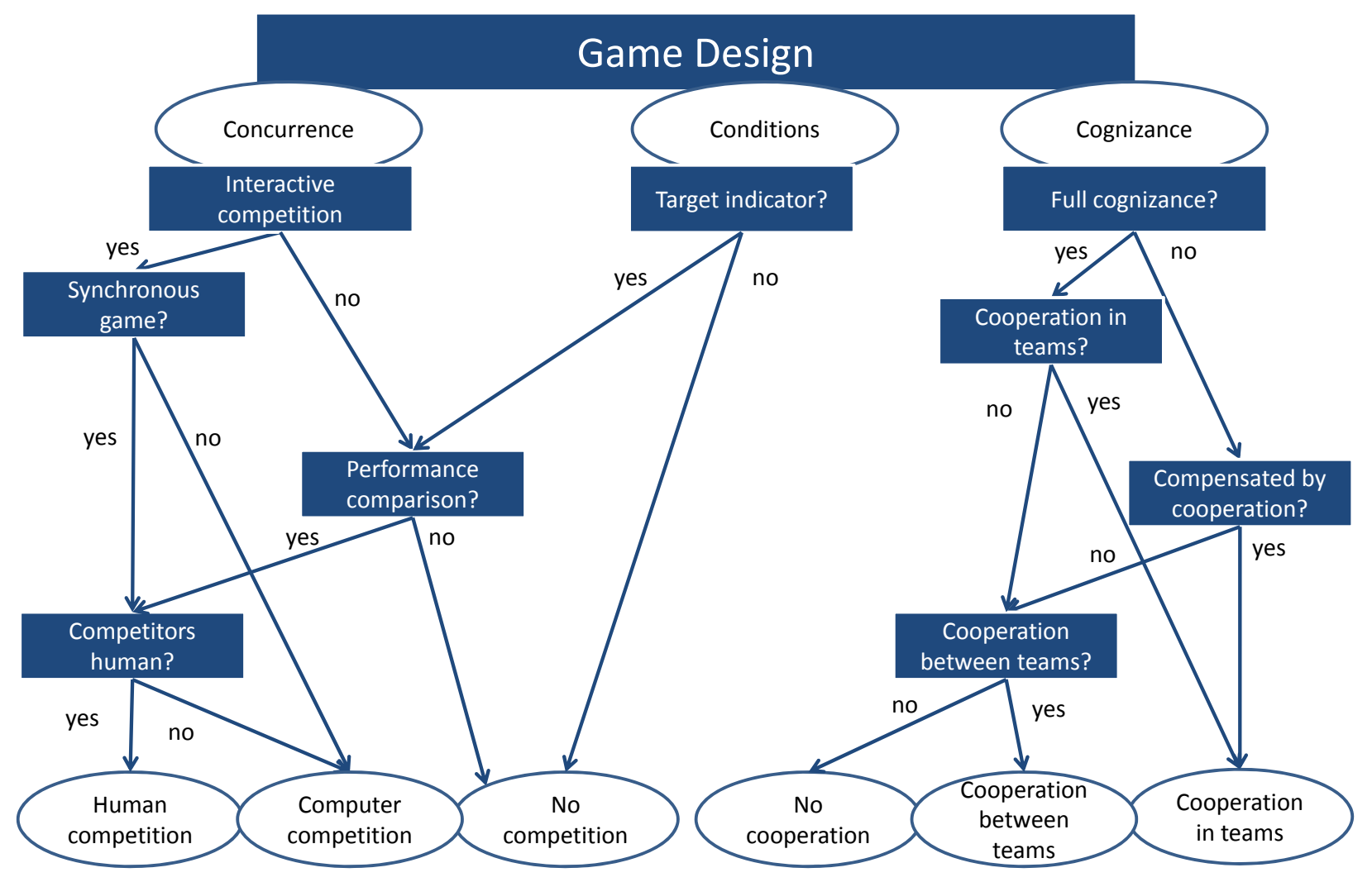

Figure 1: Aspects of Game Design in a Partial Decision Tree.

time. Alternatively, asynchronous games may be designed as tasks of analysis and exploration, presenting one or several players with a set of problems. A simulation system may be both a tool for the player and a tool for the evaluation of the player's performance in such a challenge.

Synchronous games requiring concurrence means that all players have to be available at the same time so that their actions influence each other's outcome. In the case of revenue management, such a synchronous game may consist of several players taking the role of supply agents in a participatory simulation. Each supplier's set of availabilities has a consequence for the demand other suppliers may observe. Accordingly, availability influences have to become active at the same time. The performance of players can be compared directly in the game, depending on the conditions set up for each supplier.

Depending on the simulation system used, games with and without concurrence may be conducted with all players in the same place or with distributed players communicating over a single medium. In the case of games with concurrence, synchronous timing is crucial during the game's execution stage. In games without concurrence, timing is completely optional.

\subsection{Conditions: Information and Target Indicators}

Game conditions may be phrased in terms of information. The information that is provided for players in a game is a substitute for the information available in a real-world environment. It can be used to differentiate between alternative realistic situations as well as between realistic and hypothetical situations. Several characteristics of information require consideration: its quantity, timing, distribution, form, and quality. One important piece of information should be regarded as a separate design decision, that of the target indicators used for the player's performance evaluation at the end of the game. 


\section{Cleophas}

An example for a hypothetical situation with regard to information quantity is a revenue management game where perfect information about competitor availabilities is available. Such a situation is not realistic, but the chances and challenges resulting from it may be beneficial to a discussion about competitive strategies.

The provision of information is controlled by the trainer. Timing decides whether all information is available before the game even starts or whether it is revealed only slowly over the course of game execution. Information may even be withheld to be announced only when the players' performance is evaluated.

The distribution and the form of information have to be considered as well. Do all players get the same amount and quality of information or does one player have an advantage over the others? Is information communicated in the same form in which it is available in real world situations, does it have to be acquired by the players, or is for example a new type of report introduced in the game?

As in the real world, in serious games, the quality of information can vary, it may be be misleading or flawed. Simulation systems for revenue management allow for perfect information to be available at any time if so desired. Instead of using this perfect information, the trainer may include distortions to make the game more or less realistic.

The game's target in the form of indicators to be evaluated after the game has been executed represents one important piece of information. The choice of target indicators depends on the game's focus: In a game designed to consider competitive strategies, market share may be as important or even more important than revenue. The game's focus depends on the target, the time it is announced and whether it is set by the trainer or by the players themselves.

\subsection{Cognizance: Full or Partial Responsibility}

Any revenue management game will include a set of products, for which there exists more or less segmented demand, and which can be sold at a range of different price points over the course of a sales horizon. In designing the players' cognizance, a trainer decides whether a single player is in charge of the complete range or of a subset of decision variables, allocating availability to certain products, customer segments, prices and time slices of the sales horizon.

As in the real world, analyst responsibilities may also be organized according to such subsets, the effects of cognizance can play a considerable role. Once more, this design decision influences the degree of realism of a game and opens opportunities to confront players with new or even extreme situations.

As an example of limited cognizance in airline revenue management games, players may be to analyze and influence only a single point of sale. Comparing the effects of such a policy to one of monitoring all points of sale can motivate different approaches of analysis in the real world. Alternatively, a team of players may be in charge of analyzing and influencing the availability situation for a single supplier in such a way that each player is responsible for a single point of sale. Such a set-up of distributed cognizance is likely to increase the game's aspect of cooperation as described in the next section.

\subsection{Cooperation: In Player Teams and Between Players}

Especially in games based on participatory simulations, but also in asynchronous games in the form of analysis challenges evaluated through simulations, the extent and the form of cooperation within and between teams of players can play a decisive role. The trainer is in charge of setting up the rules for such a cooperation and communicating it to the players.

Cooperation within teams of players can be important if a task - such as revenue optimization for a common supplier in a participatory game - is to be handled by several players with limited cognizance. Cooperation between individual players or teams of players may mimic the set-up of supplier revenue management alliances in the real world, which are governed by a set of strict legal and technical rules.

Depending on the game conditions, cooperation can be further enforced or complicated. When some parts of information are only available to certain players but influence other players' performance, cooperation 


\section{Cleophas}

may seem more desirable. Whether or not the overall revenue of all suppliers interacting in a market is included in the target indicators will also contribute to the role of cooperation as opposed to competition.

\subsection{Competition: Interactive or Through Comparison}

Two types of competition have to be considered as part of revenue management games. On the one hand, in participatory games, players may take on the role of supplier agents competing on a common market. In such interactive competition, the players' actions can directly influence other players' success. On the other hand, both in participatory games that are played in parallel and in asynchronous games of other types, players may be competing through performance comparison.

When the game play is participative, two types of competitors need to be differentiated. Competing agents may be controlled by human players or by the automated system. In the case of human competitors, flexible reactions and less than rational choice behavior are to be expected. In this case, which affords concurrence, the rules of communication established by the trainer can have a decisive influence on the game's outcome. When competing agents are controlled by automated algorithms implemented in the simulation system, the competitor responses are to some degree predetermined - this makes for a more consistent, but possibly less realistic competition.

By stressing the aspect of comparative performance evaluation, player competition is underlined. By emphasizing aspects of the process rather than performance, for example the idea that players discuss alternative strategies and work together toward a common goal (for example to find a robust revenue management strategy), competitive aspects of the game are relegated to the background.

\section{LAYERED GAME STAGES: BRIEFING, EXECUTION, DEBRIEFING}

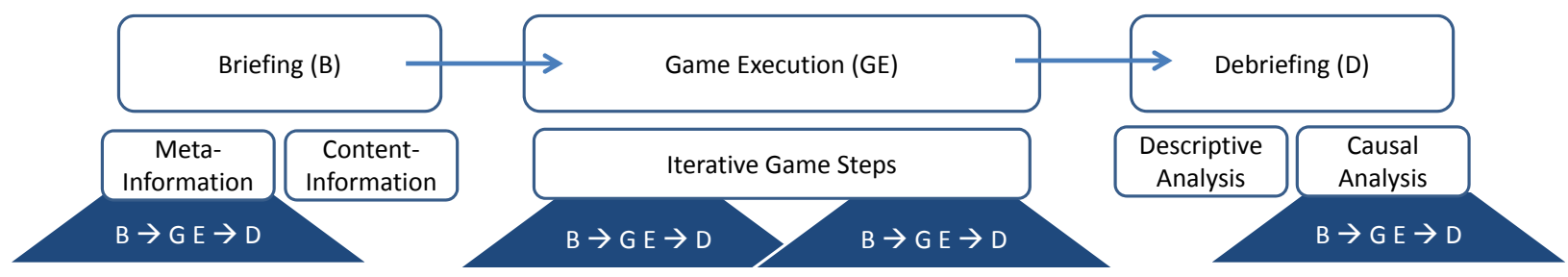

Figure 2: Serious Game Play in Layered Stages.

This paper considers serious games for revenue management as divided in three layered stages: briefing, execution and debriefing. The briefing stage prepares the game by allowing the trainer to provide players with information both about the form and the content of the game play. In the execution stage, the game itself is played and the underlying simulation is processed. In the debriefing stage, the game's outcome as well as aspects of process such as individual players' choices and deliberations can be analyzed and discussed. By considering these stages, we follow the lead of existing approaches such as (De Freitas and Oliver 2006), where the value of briefing and debriefing for the success of exploratory games based on simulations is highlighted.

As shown in Figure 2, three stages are layered: small recursions of all three stages may be implemented in each stage. For example, as part of a tutorial introducing the gaming concept, a first game may be played in the briefing stage. The execution stage may be structured in several steps, each preceded by a briefing and succeeded by a debriefing stage. Finally, in debriefing, certain parts of the game may be repeated with slight variations to test the sensitivity of the results and the robustness of player strategies.

In this part of the paper, the three stages are considered in further detail with special regard to the effects of the game design decisions met by the trainer. These effects are also illustrated by Figure 3. 


\section{Cleophas}

\begin{tabular}{|c|c|c|c|}
\hline & Briefing & Execution & Debriefing \\
\hline Concurrence & $\begin{array}{l}\text { informing about concurrence, } \\
\text { with concurrence }\end{array}$ & $\begin{array}{l}\text { synchronous or asynchronous } \\
\text { game play }\end{array}$ & $\begin{array}{l}\text { comparison over time or at one } \\
\text { time }\end{array}$ \\
\hline Conditions & providing information and target & $\begin{array}{l}\text { players act according to } \\
\text { information and target }\end{array}$ & $\begin{array}{l}\text { information completion and } \\
\text { target evaluation }\end{array}$ \\
\hline Cognizance & $\begin{array}{l}\text { assigning cognizance directly or } \\
\text { indirectly }\end{array}$ & players implement cognizance & $\begin{array}{l}\text { cognizance dissolve or } \\
\text { enforcement }\end{array}$ \\
\hline Competition & $\begin{array}{l}\text { emphasizing or downplaying } \\
\text { competition }\end{array}$ & $\begin{array}{l}\text { interactive competition } \\
\text { influences game play }\end{array}$ & $\begin{array}{c}\text { comparative or independent } \\
\text { debriefing }\end{array}$ \\
\hline Cooperation & $\begin{array}{l}\text { Announcing rules and targets of } \\
\text { cooperation }\end{array}$ & communication for cooperation & common or individual evaluation \\
\hline
\end{tabular}

Figure 3: Effects of Design Aspects in Three Stages.

\subsection{Briefing: Preparing Revenue Management Games}

Two types of information have to be conveyed in the briefing stage of serious games for revenue management: Meta-information about the game's set-up and content information as described when considering the aspect "conditions". The meta-information that the trainer needs to communicate to the players depends both on design decisions as described in Section 3 and on the players previous experience with this type of game.

In this stage, information taken from previous simulations executed without player participation or previous participative games may be recaptured. The transfer of this information can take place outside of the simulation system or aided by the data analysis component of such a system.

Concurrence: If the game is played in concurrence, it is possible that all players are in the briefing stage at the same time. If the game is participatory, the trainer may use the briefing stage to inform players about the degree to which they influence one-an-others' success on the market. Do all suppliers offer completely substitutable or complementary products? Are there certain demand segments that can be expected to prefer one supplier over another? Do all players or only some players compete on the same market?

Conditions: The briefing stage is the stage in which the initial information needs to be communicated to the players, thereby implementing the game's conditions. This stage is the first opportunity for the trainer to provide the designated quantity and quality of information in the designated form and distribution according to the planned timing. If target indicators are to be announced before the analysis phase, this is also due during the briefing stage. If the players are to decide on their own performance indicators, they should do so now to avoid ex-post bias.

Cognizance: Player's cognizance can be prepared in the briefing stage by providing different sets of information and target indicators to different players. If players are to consciously ignore certain sets of products or sales channels, they need to be told so from the start.

Cooperation: If the game is to include the opportunity for cooperation, the trainer may choose to inform the players about it in the briefing stage. Teams and rules for cooperation can be set up now, the players may be assigned to or made to pick alliance partners.

Competition: As described previously, the game's aspect of competition can be emphasized or downplayed by the trainer at several times during the game. Highlighting competitive aspects can entail announcing the comparison of certain indicators and the players' opportunity to actively influence their competitors' success in the briefing stage. A less competitive game may be realized by initializing a common discussion and possibly even the organization of how different strategies are to be evaluated by different players. 


\section{Cleophas}

\subsection{Execution: Observing and Controlling Revenue Management Games}

The execution phase may include more than a single game step. Information analysis and player actions influencing the revenue management strategy as well as the processing of these influences by the simulation systems may be repeated in iterative steps. This can lead to a number of layered stages within this stage as illustrated by Figure 2 .

Execution is the step that most relies on the use of a simulation system, as the players' decisions and their consequences have to be realized and computed in this system. If layered stages of briefing and debriefing are involved, these may take the form of data analysis aided by the simulation system.

Concurrence: Whether or not a game is played in concurrence significantly influences its execution stage. If the game is played synchronously, the execution stage has to include a common timing for all players, enabling direct and indirect interaction between the players. Participatory games where all participants are to take on the role of suppliers competing on a common market have to be concurrent.

Conditions: During the game's execution stage, further information may be made available to the players. The players' target indicators determine the way in which the game is played, its atmosphere and focus.

Cognizance: The players' cognizance as assigned by the trainer is implemented during the game execution. Consciously (as directed by the trainer) or unconsciously (based on their information or lack thereof), players will focus on certain decision variables and ignore others.

Cooperation: If the game allows for cooperation, this is realized mostly in the execution stage. Any rules regarding cooperation have to be implemented now, with other aspects such as concurrence, conditions, cognizance and the existence of competition influencing the feasible forms of cooperation.

Competition: If players compete directly by representing suppliers on a common market, the intermediate outcomes of each step of the game may represent a type of indirect communication. If competition is controlled by the computer, the trainer may use observations collected during early parts of the game's execution to parametrize the automated system's reaction.

\subsection{Debriefing: Analyzing Revenue Management Games}

As illustrated by Figure 2, the debriefing phase can be used for both descriptive and causal analysis. As part of descriptive analysis, result indicators may be assessed and compared for all players. As part of causal analysis, the causal factors that influenced each player's results may be analyzed.

The debriefing phase can be governed by the trainer presenting the game's results or by the player's autonomous analysis and discussion of result indicators. For this purpose, the simulation system can serve as the main tool; alternatively, the analysis can be based on independent tools - from spreadsheets to pen and paper - using data exported from the simulation.

Concurrence: If the game was played in concurrence, debriefing can occur for all players simultaneously. If the game was played asynchronously, comparative analysis is only feasible after all players are finished. In the case of players interacting in a participatory game, causal analysis can include discussing how and why one player's actions lead to an player's success or lack thereof.

Conditions: If incomplete or distorted information was provided during briefing and execution, the debriefing phase may be used to correct faulty assumptions and complete the player's knowledge about the actual conditions of the game. The debriefing phase can use on one of the advantages of simulations as opposed to real-world situations: The trainer is able to trace back each step and know about all influencing factors included in the system. If the game had clearly defined target indicators, players may be evaluated accordingly.

Cognizance: Any limitations with regard to cognizance may be dissolved or emphasized during debriefing. A game that was set up to show how limited cognizance can limit or improve the player's success can be debriefed by comparing the player's results to those of a game where cognizance was assigned differently. 


\section{Cleophas}

Cooperation: If the game included an element of cooperation, this can be further emphasized by aggregating the target indicators over all cooperating players, evaluating teams rather than individuals. Individual results may be compared with team results to isolate and measure the effect of cooperation.

Competition: As discussed with regard to concurrence, the causal analysis of players' performance has to take into account competitor reactions in the case of interactive competition. Finally, the aspect of competition can be modulated by focusing on absolute or comparative performance indicators in this stage.

\section{CONCLUSION AND OUTLOOK}

By considering the literature regarding revenue management simulation systems and the implementation of serious games, this paper motivated the design of serious games for revenue management training and strategy development. By emphasizing revenue management challenges such as uncertain demand, direct competition and complex algorithms, factors underlining the applicability of serious games in this field were highlighted. In this regard, simulation systems are well-suited to complement tutoring by allowing players to directly experience the effects of uncertain demand and to interact with realistic representations of complex revenue management systems without the risk and cost associated to real-world experiments.

The framework introduced in this paper regarded serious games for revenue management as structured according to three layered stages and five design aspects. Decisions with regard to the concurrence, conditions, cognizance, cooperation and competition implemented in games were first listed and then considered as they play out in the briefing, execution and debriefing stages.

The presented framework is intended to evaluate future alternative designs of serious games aiming to improve revenue management understanding and strategy evaluation. Given specific objectives, it is not yet clear how a serious game should ideally be designed. By realizing alternatives according to the aspects and stages presented and evaluating the outcome, this research gap may be attended to in the future.

The resulting opportunities for future research can be illustrated using two examples. When serious games are implemented for the development of competitive revenue management strategies, this may be done by including interactive human competition or by relying on competition modeled through automated simulation agents. Both approaches have their own benefits and lacks and may be evaluated only based on actual experimentation. With regard to training for revenue management, new concepts may be conveyed both in participatory simulations or in individual analysis games. Deliberate experimentation may provide insight into the effectiveness of both approaches.

Further potential lies in the application of the framework presented here to other disciplines. As part of such an effort, existing serious games could be categorized with regard to the design decisions described. Such an extension would exceed the scope and focus of this paper and therefore has to be delegated to future research.

\section{ACKNOWLEDGMENTS}

The author would like to thank Deutsche Lufthansa AG for making the REMATE revenue management simulation system available, providing the opportunity to set-up, conduct and observe revenue management games and enabling continuous contact with revenue management practitioners.

\section{REFERENCES}

Abdelghany, A., and K. Abdelghany. 2007. "Evaluating airlines ticket distribution strategies: a simulationbased approach". International Journal of Revenue Management 1 (3): 231-246.

Abdelghany, A., and K. Abdelghany. 2008. "A micro-simulation approach for Airline Competition Analysis and Demand Modelling". International Journal of Revenue Management 2 (3): 287-306.

Belobaba, P., and J. Wilson. 1997. "Impacts of yield management in competitive airline markets". Journal of Air Transport Management 3 (1): 3-9. 


\section{Cleophas}

Charsky, D. 2010. "From edutainment to serious games: A change in the use of game characteristics". Games and Culture 5 (2): 177-198.

Cleophas, C. 2012a. "Assessing Multi-Agent Simulations - Inspiration through Application.”. In Advances in Intelligent and Soft Computing 156: Highlights on Practical Applications of Agents and Multi-Agent Systems - 10th International Conference on Practical Applications of Agents and Multi-Agent Systems, edited by J. B. Pérez, M. A. Sánchez, P. Mathieu, J. M. C. Rodríguez, E. Adam, A. Ortega, M. N. Moreno, E. Navarro, B. Hirsch, H. Lopes-Cardoso, and et al., 163 - 170. Springer.

Cleophas, C. 2012b. "Multi-agent modelling for revenue management". Journal of Revenue \& Pricing Management 11 (2): 240-242.

Cleophas, C., M. Frank, and N. Kliewer. 2009. "Simulation-based key performance indicators for evaluating the quality of airline demand forecasting". Journal of Revenue and Pricing Management 8 (4): 330-342.

Currie, C., and I. Rowley. 2010. "Consumer behaviour and sales forecast accuracy: What's going on and how should revenue managers respond\&quest". Journal of Revenue \& Pricing Management 9 (4): 374-376.

De Freitas, S., and M. Oliver. 2006. "How can exploratory learning with games and simulations within the curriculum be most effectively evaluated?". Computers \& Education 46 (3): 249-264.

Frank, M., M. Friedemann, and A. Schröder. 2008. "Principles for simulations in revenue management". Journal of Revenue \& Pricing Management 7 (1): 7-16.

Gilbert, G. 2008. Agent-based models. Sage Publications, Inc.

Gorin, T., D. Walczak, P. Bartke, and M. Friedemann. 2012. "Incorporating cancel and rebook behavior in revenue management optimization". Journal of Revenue \& Pricing Management. Forthcoming.

Halter, E. 2006. From Sun Tzu to xbox: War and video games. PublicAffairs.

Hoang, P. 2007. "The future of revenue management and pricing science". Journal of Revenue \& Pricing Management 6 (2): 151-153.

Hopperstad, C. 1995. "Potential Application of the Boeing Passenger Origin-Destination Simulator (PODS) to Yield Management System Planning". Technical report, Revision C. Boeing Commercial Airplane Group.

Johnson, W. 2007. "Serious use of a serious game for language learning". In Proceeding of the 2007 conference on Artificial Intelligence in Education: Building Technology Rich Learning Contexts That Work, edited by R. Luckin, K. Koedinger, and J. Greer, 67-74. IOS Press.

Kato, P. 2010. "Video games in health care: Closing the gap.". Review of General Psychology 14 (2): 113. Law, A., and D. Kelton. 2000. "Simulation modeling and analysis".

Mathieu, P., D. Panzoli, and S. Picault. 2011. "Format-store: a multi-agent based approach to experiential learning". In Games and Virtual Worlds for Serious Applications (VS-GAMES), 2011 Third International Conference on, edited by F. Liarokapis, D. A., and V. V., 120-127. IEEE.

McMahon-Beattie, U., and I. Yeoman. 2011. Revenue Management: A Practical Pricing Perspective. London, UK: Palgrave Mamillan.

Michael, D., and S. Chen. 2005. Serious games: Games that educate, train, and inform. Muska \& Lipman/Premier-Trade.

Mukhopadhyay, S., S. Samaddar, and G. Colville. 2007. "Improving Revenue Management Decision Making for Airlines by Evaluating Analyst-Adjusted Passenger Demand Forecasts". Decision Sciences 38 (2): 309-327.

Puschel, T., F. Lang, C. Bodenstein, and D. Neumann. 2010. "A Service Request Acceptance Model for Revenue Optimization-Evaluating Policies Using a Web Based Resource Management Game". In System Sciences (HICSS), 2010 43rd Hawaii International Conference on, edited by R. H. J. Sprague, 1-10. IEEE.

Ramanath, A., and N. Gilbert. 2004. "The design of participatory agent-based social simulations". Journal of Artificial Societies and Social Simulation 7 (4).

Talluri, K., and G. van Ryzin. 2004. Theory and Practice of Revenue Management. Kluwer, Boston. 


\section{Cleophas}

\section{AUTHOR BIOGRAPHIES}

CATHERINE CLEOPHAS studied business computing with an emphasis on operations research and decision support at the University of Paderborn, Germany. From 2006 to 2009 she worked on her doctoral thesis as a Lufthansa fellow at the graduate school of dynamic intelligent systems. In 2009, she received her $\mathrm{PhD}$ for her work on simulation-based evaluation of forecasts for revenue management. Until April 2011, she worked as a consultant in the revenue management development and simulation team at Deutsche Lufthansa. Since April 2011, she holds a position as assistant professor at the department information systems at Freie Universität Berlin. She is especially interested in simulation-based method evaluation and decision support as well as in the validation and calibration of market simulations. Her email address is catherine.cleophas@fu-berlin.de. 